\title{
MicroRNA-92a promotes metastasis of nasopharyngeal carcinoma by targeting the PTEN/AKT pathway
}

This article was published in the following Dove Press journal:

OncoTargets and Therapy

16 June 2016

Number of times this article has been viewed

\author{
Haixiong Zhang' \\ Hui Cao' \\ Dadao Xu' \\ Kang Zhu ${ }^{2}$ \\ 'Department of Otorhinolaryngology, \\ Head and Neck Surgery, the \\ First Affiliated Hospital of Xi'an \\ Medical College, ${ }^{2}$ Department of \\ Otorhinolaryngology, Head and Neck \\ Surgery, the Second Affiliated Hospital \\ of Xi'an Jiaotong University, Xi'an, \\ People's Republic of China
}

\begin{abstract}
MicroRNAs have been confirmed to be a group of important regulators during the pathogenesis of nasopharyngeal carcinoma (NPC). This study confirmed that the expression of microRNA-92a (miR-92a) was significantly upregulated in NPC as compared to noncancerous nasopharyngeal epithelial tissues. Furthermore, high expression of miR-92a was observed in all NPC cell lines, especially in high metastatic cell lines. Clinical analysis indicated that high expression of miR-92a was associated with adverse clinicopathological features including the advanced tumor-node-metastasis stage and distant metastasis, and conferred poor prognosis of patients. In vitro assays showed that miR-92a overexpression potentiated the migration and invasion of 6-10B cells, and miR-92a silencing reduced the number of migrated and invaded 5-8F cells. Phosphatase and tensin homolog (PTEN) was confirmed as a direct downstream target of miR-92a in NPC cells. Otherwise, alteration of miR-92a expression regulated PTEN/ AKT pathway in NPC cells. Mechanistically, miR-92a exerted its promoting effects on the metastatic behaviors of NPC cells through suppressing PTEN/AKT pathway. Taken together, this study demonstrates that miR-92a is a promising prognostic biomarker for patients with NPC, and may be a potential therapeutic target to prevent the metastasis of NPC.
\end{abstract}

Keywords: microRNA-92a, nasopharyngeal carcinoma, PTEN, signaling pathway, tumor metastasis, non-coding RNA, head and neck neoplasms

\section{Introduction}

Nasopharyngeal carcinoma (NPC), which predominantly occurs in the southern part of the People's Republic of China, is a malignant epithelial tumor of the head and neck. ${ }^{1-3}$ Although remarkable progress has been made in the therapeutic strategy of NPC, the long-term survival of patients with NPC is still unsatisfactory. The major reasons for the poor prognosis of patients with NPC include local recurrence and systemic metastasis. ${ }^{4,5}$ Therefore, it is of great importance to clarify the molecular mechanisms for the metastatic processes of NPC cells, which may facilitate the development of novel therapeutic targets for NPC.

MicroRNAs (miRNAs) is a group of short noncoding RNAs, which regulate gene expression through mRNA cleavage and/or translation inhibition by binding to the $3^{\prime}$-untranslated region ( $3^{\prime}$-UTR) of the targeted mRNA. ${ }^{6}$ It can modulate various cellular processes including proliferation, apoptosis, movement, and differentiation. ${ }^{7-9}$ And numerous studies have demonstrated that aberrant expression or dysfunction of miRNAs plays critical roles in the initiation and progression of various human malignancies. ${ }^{10-12}$ 
Among numerous miRNAs, microRNA-92a (miR-92a), which belongs to the cluster of miR-17-92, has been found to be a novel cancer-related miRNA. Abnormal expression and dysfunction of miR-92a have been found in hepatocellular carcinoma (HCC), ${ }^{13}$ lung cancer, ${ }^{14}$ colorectal cancer, ${ }^{15}$ breast cancer, ${ }^{16}$ and cervical cancer. ${ }^{17}$ Increased level of miR-92a expression was confirmed in HCC, ${ }^{13}$ lung cancer, ${ }^{14}$ and colorectal cancer ${ }^{15}$ and was correlated with poor prognosis of patients with cancer. These results suggest that miR-92a plays an oncogenic role in human cancers. However, studies of ovarian cancer ${ }^{18}$ and breast cancer ${ }^{16}$ showed that the expression of miR-92a was downregulated, indicating a tumor-suppressive function of miR-92a. These studies demonstrate that the exact biological role of miR-92a varies in different cancers. However, the expression and functional role of miR-92a in NPC have never been examined before.

In this study, we found that miR-92a expression was elevated in NPC tissues and cells, and increased expression of miR-92a was associated with poor prognosis of patients with NPC. Functionally, we found that miR-92a could promote the migration and invasion of NPC cells. Furthermore, phosphatase and tensin homolog (PTEN)/AKT pathway was identified to be a direct functional downstream target of miR-92a.

\section{Materials and methods Clinical tissues and cell culture}

Eighty six freshly frozen NPC specimens and 20 normal nasopharyngeal epithelium specimens were obtained from the Department of Otorhinolaryngology, Head and Neck Surgery, the Second Affiliated Hospital of Xi'an Jiaotong University during January 2006 to December 2009. None of the patients received radiotherapy or chemotherapy before biopsy. Written informed consent was obtained from every patient. Table 1 shows the demographic features and clinicopathologic data. The ethics committee of Xi'an Jiaotong University approved all protocols involving patients' samples according to the Declaration of Helsinki (as revised in Tokyo 2004).

The human NPC cell lines CNE-1, CNE-2, 5-8F, and 6-10B were cultured in Roswell Park Memorial Institute1640 (Invitrogen, Carlsbad, CA, USA) supplemented with $5 \%$ fetal bovine serum (Gibco, Grand Island, NY, USA), $100 \mathrm{U} / \mathrm{mL}$ penicillin, and $100 \mathrm{U} / \mathrm{mL}$ streptomycin. The human immortalized nasopharyngeal epithelial cell lines NP69 were cultured in serum-free medium (Invitrogen) supplemented with growth factors (Gibco). All cells were maintained in a humidified chamber supplemented with 5\% carbon dioxide.
Table I Correlation between the clinicopathologic characteristics and expression of miR-92a in NPC

\begin{tabular}{|c|c|c|c|c|}
\hline \multirow[t]{2}{*}{ Characteristics } & \multirow{2}{*}{$\begin{array}{l}\text { Total no of } \\
\text { patients, } \\
n=86\end{array}$} & \multicolumn{2}{|c|}{ No of patients } & \multirow[t]{2}{*}{$P$-value } \\
\hline & & $\begin{array}{l}\text { miR-92a } \\
\text { high }\end{array}$ & $\begin{array}{l}\text { miR-92a } \\
\text { low }\end{array}$ & \\
\hline \multicolumn{5}{|l|}{ Age (years) } \\
\hline$\leq 45$ & 42 & 20 & 22 & 0.666 \\
\hline$>45$ & 44 & 23 & 21 & \\
\hline \multicolumn{5}{|l|}{ Sex } \\
\hline Male & 63 & 30 & 33 & 0.465 \\
\hline Female & 23 & 13 & 10 & \\
\hline \multicolumn{5}{|l|}{ WHO type } \\
\hline I+II & 9 & 3 & 6 & 0.483 \\
\hline III & 77 & 40 & 37 & \\
\hline \multicolumn{5}{|l|}{ TNM stage } \\
\hline I+II & 28 & 8 & 20 & $0.006 *$ \\
\hline III+IV & 58 & 35 & 23 & \\
\hline \multicolumn{5}{|l|}{ Distant metastasis } \\
\hline No & 68 & 29 & 39 & $0.008 *$ \\
\hline Yes & 18 & 14 & 4 & \\
\hline
\end{tabular}

Note: *Statistically significant.

Abbreviations: miR-92a, microRNA-92a; NPC, nasopharyngeal carcinoma; TNM, tumor-node-metastasis; WHO, World Health Organization.

\section{Quantitative real-time polymerase chain reaction}

The RNA from clinical specimens and NPC cells were extracted using Trizol (Invitrogen) following the manufacturer's instruction. The TaqMan miRNA reverse transcription kit (Applied Biosystems, Foster City, CA, USA) and TaqMan human MiRNA assay kit (Applied Biosystems) were employed for the polymerase chain reaction (PCR) amplification of the miR-92a and U6. Primers against miR92a (HmiRQP0832) and U6 (HmiRQP9001) were purchased from Genecopoeia (Guangzhou, People's Republic of China). The relative expression of miR-92a was shown as fold difference relative to U6.

\section{Cell transfection}

The miR-92a mimics, miR-92a inhibitor, and the corresponding control vectors were bought from Genecopoeia (Guangzhou). The PTEN-specific small interfering RNA (siRNA) (\#25638), and PTEN expressing vector (\#28298) were obtained from Addgene (Cambridge, MA, USA). The $5 \times 10^{5} \mathrm{NPC}$ cells ( $6-10 \mathrm{~B}$ cells or $5-8 \mathrm{~F}$ cells) were seeded in $60 \mathrm{~mm}$ plates $18-24$ hours before transfection. The 5-10 $\mu \mathrm{L}$ lentiviral particles carrying miR-92a expressing vector $\left(10^{8} \mathrm{TU} / \mathrm{mL}\right)$ were transfected into $6-10 \mathrm{~B}$ cells to increase the expression level of miR-92a; 5-10 $\mu \mathrm{L}$ lentiviral particles carrying miR-92a inhibitor were employed for suppressing the expression level of miR-92a in 5-8F cells. Following the 
protocols of Lipofectamine 2000 (Invitrogen), PTEN siRNA was transfected into 5-8F cells for suppression of miR-92a while PTEN overexpression vector was transfected into 6-10B cells for overexpressing miR-92a.

\section{Western blot}

Cellular proteins were collected using the radioimmunoprecipitation assay lysis buffer (Santa Cruz Biotechnology, Inc., Santa Cruz, CA, USA), and protein concentration was measured using the bicinchoninic acid kit (Pierce, Rockford, IL, USA). Thirty microgram protein of each sample was separated by sodium dodecyl sulfate-polyacrylamide gel electrophoresis and transferred to polyvinylidene difluoride membrane. The blots were incubated with the following primary antibodies overnight: PTEN (1:1,500, \#9552, Cell Signaling Technologies, Danvers, MA, USA), AKT (1:1,000, sc-8312, Santa Cruz Biotechnology, Inc.), p-AKT (1:1,000, sc-33437, Santa Cruz Biotechnology, Inc.), and glyceraldehyde 3-phosphate dehydrogenase (1:1,500, sc-32233, Santa Cruz Biotechnology, Inc.). Then, the membranes were incubated with antimouse or antirabbit secondary antibodies (1:10,000; Bio-Rad Laboratories Inc., Hercules, CA, USA), and the signals were detected using the Bio-Rad gel imaging system (Bio-Rad Laboratories Inc.).

\section{Transwell assays}

Transwell assays were performed to evaluate the migratory and invasive ability of NPC cells. NPC cells were transfected with miR-92a mimics or inhibitor in six-well plates before seeding into the transwell chambers. The cells were resuspended in serum-free medium and were seeded into plates with $8 \mu \mathrm{m}$ BioCoat control inserts (BD Biosciences, Bedford, MA, USA). Serum-containing medium ( $20 \%$ fetal bovine serum) was used in the lower chamber as the attractant. Regarding the invasion assay, each upper chamber was coated with a mixture of Dulbecco's Modified Eagle's Medium and Matrigel (Becton-Dickinson Labware, Bedford, MA, USA) at a ratio of $6: 1 ; 24$ hours after cell seeding, NPC cells on the upper surface of the filter were removed with cotton swabs. Then, the migrated or invaded cells in the lower surface were stained with $0.1 \%$ crystal violet, and the cell numbers were obtained from ten different fields of the lower surface of the filter. Three independent experiments were performed.

\section{Luciferase reporter assay}

Wild-type (wt) 3'-UTR of PTEN and the mutated sequence were inserted into the pGL3 control vector (Promega Corporation, Madison, WI, USA) to construct wt PTEN-3'-UTR vector and mutant PTEN-3'-UTR vector, respectively. For luciferase reporter assay, 6-10B cells were transfected with the corresponding vectors; 48 hours after transfection, the dual-luciferase reporter assay system (Promega, Shanghai, the People's Republic of China) with the luminometer (Promega) were used to measure the luciferase activity. Three independent experiments in duplicate were performed to obtain the results of luciferase activity.

\section{Statistical analysis}

Statistical analysis was performed with the GraphPad Prism 5 software (GraphPad Software, Inc., La Jolla, CA, USA). The Pearson's chi-square test, the Kaplan-Meier plot and log-rank test, and the Student's $t$-test were performed in this study. $P<0.05$ was considered to indicate a statistically significant result.

\section{Results}

\section{The expression of miR-92a is increased in NPC tissues and cells}

The expression levels of miR-92a in 86 NPC tissues and 20 normal tissues were measured by quantitative real-time polymerase chain reaction. We found that the level of miR-92a expression in NPC tissues was significantly higher than that in normal tissues $(P<0.05$, Figure 1A). Next, we analyzed the expression of miR-92a in four NPC cell lines (5-8F, 6-10B, CNE1, and CNE2) and the nasopharyngeal epithelial cell line NP69. The expression of miR-92a was obviously upregulated in all NPC cell lines as compared to NP69 $(P<0.05$, Figure 1B). These data suggest that miR-92a probably plays an oncogenic role in the initiation and progression of NPC.

\section{Increased level of miR-92a is correlated with the adverse clinicopathological features and poor prognosis of patients with NPC}

After confirming the aberrant elevated expression of miR92a in NPC tissues, we examined whether abnormal expression of miR-92a was associated with the survival of patients with NPC. Based on the median level of miR-92a expression, we divided the patients with NPC into two groups: miR-92a low expression group $(n=43)$ and miR-92a high expression group $(n=43)$. Then, the association between clinicopathologic features and miR-92a expression was analyzed. As presented in Table 1, high level of miR-92a was evidently associated with tumor-node-metastasis stage $(P=0.006)$ and distant metastasis $(P=0.008)$. Furthermore, 

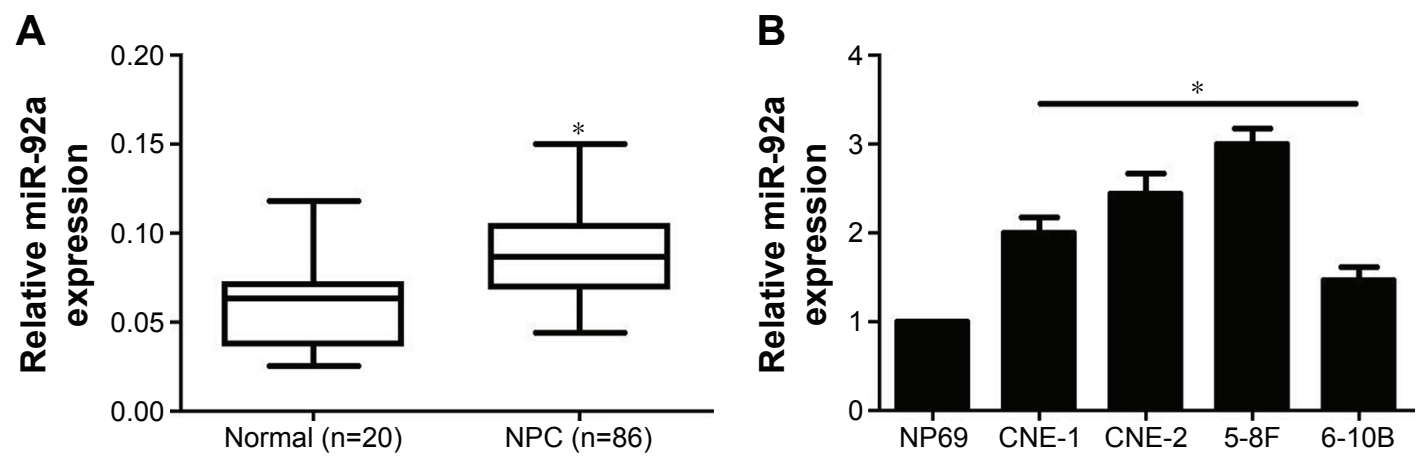

Figure I The expression levels of miR-92a in NPC tissues and cells.

Notes: The differences of miR-92a expression between (A) NPC tissues and normal tissues, (B) four different NPC cell lines (CNE-I, CNE-2, 5-8F, and 6-I0B), and normal human nasopharyngeal epithelial cell line (NP69). $* P<0.05$.

Abbreviations: miR-92a, microRNA-92a; NPC, nasopharyngeal carcinoma.

the Kaplan-Meier analysis showed that patients with high expression of miR-92a had significant decreased overall survival $(P=0.047$, Figure $2 \mathrm{~A})$ and disease-free survival ( $P=0.039$, Figure 2B). Otherwise, multivariant Cox regression analysis demonstrated that miR-92a expression was an independent prognostic marker for predicting both overall survival and disease-free survival in patients with NPC ( $P=0.012$ and $P=0.009$, respectively, Table 2$)$. These data indicate that miR-92a can potentially serve as an effective biomarker for the prognosis of patients with NPC.

\section{miR-92a potentiates the migratory and invasive ability of NPC cells}

Since local recurrence and systemic metastasis are the culprits for the poor prognosis of patients with NPC, we examined whether miR-92a could modulate the metastatic ability of NPC cells. The detection of miR-92a level in NPC cell lines indicated that miR-92a showed the highest level in $5-8 \mathrm{~F}$ cells and lowest level in $6-10 \mathrm{~B}$ cells. Therefore, $5-8 \mathrm{~F}$ cells were chosen for the loss-of-function experiments and 6-10B cells for gain-of-function experiments. The miR-92a mimics were transfected into 6-10B cells, and the expression level of miR-92a in 6-10B cells was significantly increased after transfection $(P<0.05$, Figure 3A). Then, we examined the alteration of the migration and invasion of 6-10B cells after miR-92a overexpression. The results of transwell assays showed that overexpression of miR-92a significantly increased the migration and invasion $(P<0.05$, respectively, Figure 3B) of NPC cells. To rule out the possibility that the increased migration and invasion of NPC cells is due to the altered cell survival, we performed 5-bromo-2'-deoxyuridine assays to determine cell proliferation; 24 hours after miR-92a mimics transfection, the results showed that miR-92a overexpression did not significantly affect $6-10 \mathrm{~B}$ cell proliferation $(P>0.05)$. To further confirm the promoting effects of miR-92a on the migration

\section{A}

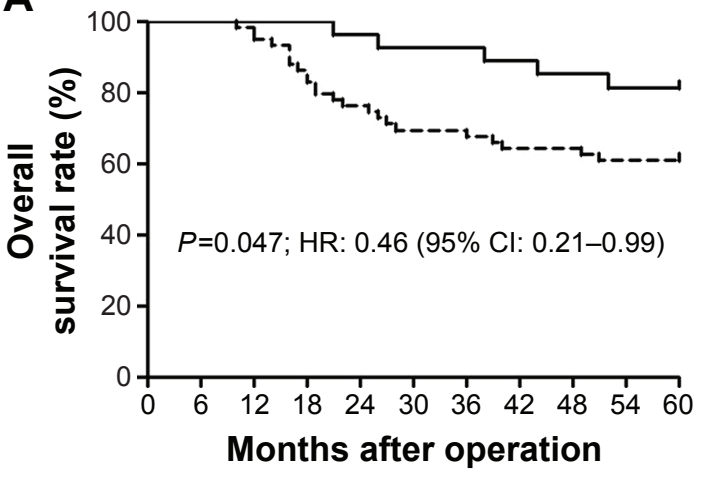

B

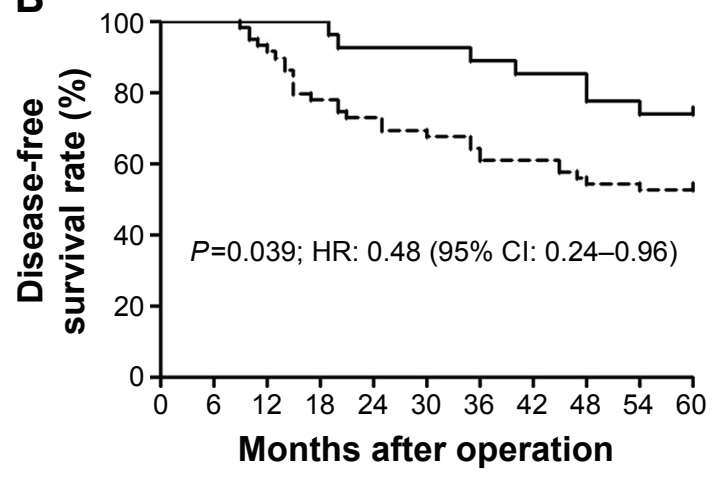

土 miR-92a low expression $(n=43) \quad$ - + . miR-92a high expression $(n=43)$

Figure 2 Elevated level of miR-92a was associated with poor prognosis of patients with NPC.

Notes: Compared with those with low miR-92a level, patients with high miR-92a level had significant shorter (A) overall survival and (B) disease-free survival. Abbreviations: $\mathrm{Cl}$, confidence interval; HR, hazard ratio; miR-92a, microRNA-92a; NPC, nasopharyngeal carcinoma. 
Table 2 Multivariate Cox regression analysis of overall survival duration

\begin{tabular}{|c|c|c|c|c|c|c|}
\hline \multirow[t]{2}{*}{ Variables } & \multicolumn{3}{|c|}{ Overall survival } & \multicolumn{3}{|c|}{ Disease-free survival } \\
\hline & HR & $95 \% \mathrm{Cl}$ & $P$-value & HR & $95 \% \mathrm{Cl}$ & $P$-value \\
\hline TNM stage & 2.067 & $1.130-3.783$ & $0.019 *$ & 3.434 & I.349-8.746 & $0.010 *$ \\
\hline $\begin{array}{l}\text { Distant } \\
\text { metastasis }\end{array}$ & 4.421 & 2.209-8.847 & $0.000^{*}$ & 2.606 & $1.245-5.453$ & $0.011 *$ \\
\hline $\begin{array}{l}\text { miR-92a } \\
\text { expression }\end{array}$ & 2.004 & $1.182-3.413$ & $0.012^{*}$ & 2.930 & I.443-5.972 & $0.009 *$ \\
\hline
\end{tabular}

Note: *Statistically significant.

Abbreviations: $\mathrm{Cl}$, confidence interval; $\mathrm{HR}$, hazard ratio; miR-92a, microRNA-92a; TNM, tumor-node-metastasis.

and invasion of NPC cells, we inhibited the expression of miR-92a in 5-8F cells with miR-92a inhibitor. Transfection of miR-92a inhibitor significantly downregulated the level of miR-92a in 5-8F cells $(P<0.05$, Figure $3 \mathrm{C})$, and led to significant decreased ability of migration and invasion $(P<0.05$, respectively, Figure 3D) of 5-8F cells. And the cell proliferation was also not significantly affected by transfection of the miR-92a inhibitor $(P>0.05)$. These results demonstrate that miR-92a exerts its oncogenic role in NPC by potentiating the migratory and invasive ability of NPC cells.

\section{PTEN is a downstream target of miR-92a} in NPC cells

To further elucidate the underlying molecular mechanisms involved in the oncogenic role of miR-92 in NPC cells, we used two publicly available databases TargetScan 7.0 (http://www.targetscan.org/) and miRanda (http://www.microrna.org/) to search for the potential downstream targets of miR-92a. The data of these two public databases showed that PTEN, a canonical tumor suppressor in human cancer, ${ }^{19}$ was potentially a downstream target of miR92a. As suggested in Figure 4A, the complementary sequence of miR-92a was found in the $3^{\prime}$-UTR of PTEN mRNA. To confirm that miR-92a could interact with 3 '-UTR of PTEN through the complementary sequence, the luciferase reporter assay was performed. The results showed that forced expression of miR-92a significantly decreased the luciferase activity of PTEN with wt 3'-UTR $(P<0.05$, Figure 4B), but did not influence that of PTEN with mutant 3 '-UTR (Figure 4B). We further examined whether miR-92a could modulate the expression of PTEN in NPC cells. The results of Western blot showed that overexpression of miR-92a in 6-10B cells significantly reduced the expression of PTEN $(P<0.05$, Figure 5A). Accordingly,
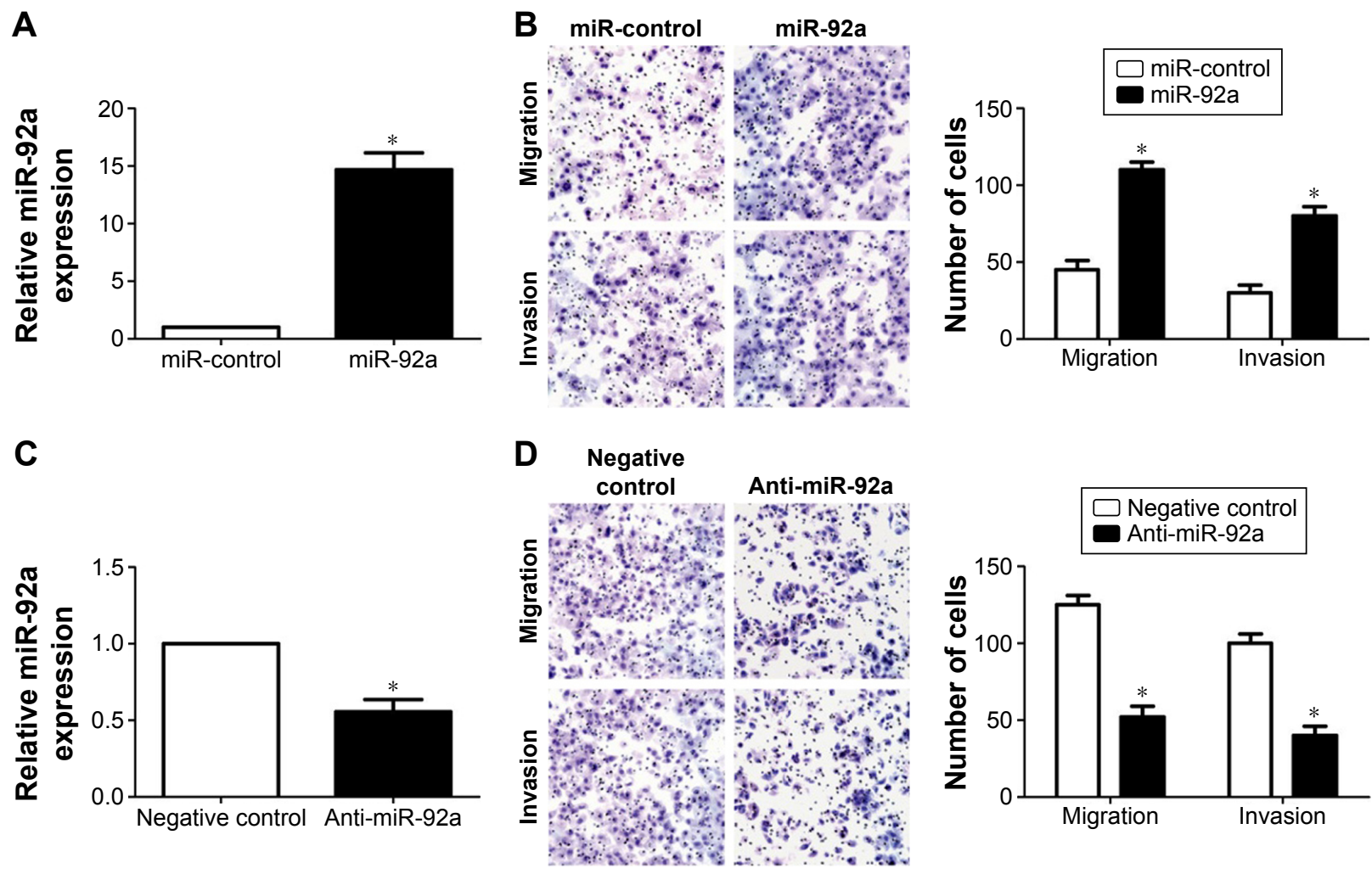

Figure 3 miR-92a promotes the migration and invasion of NPC cells.

Notes: $(\mathbf{A})$ Transfection of miR-92a mimics significantly increased the expression level of miR-92a in $6-10 B$ cells; $n=3$ independent repeats with similar results; $* P<0.05$. (B) Overexpression of miR-92a significantly promoted the migration and invasion of 6-10B cells. $n=3$ repeats with similar results; $* P<0.05$. (C) miR-92a inhibitor significantly downregulated the level of miR-92a in $5-8 \mathrm{~F}$ cells; $n=3$ independent repeats with similar results; $* P<0.05$. (D) Downregulation of miR-92a significantly inhibited the migration and invasion of $5-8 \mathrm{~F}$ cells; $\mathrm{n}=3$ repeats with similar results; $* \mathrm{P}<0.05$.

Abbreviations: miR-92a, microRNA-92a; NPC, nasopharyngeal carcinoma. 
A

\begin{tabular}{|c|c|c|}
\hline Hsa-miR-92a-3p & $3^{\prime}$ & UGUCCGGCCCUGUUCACGUUAU \\
\hline$(2,859-2,866)$ & & |||||| $\mid$ \\
\hline wt PTEN-3'-UTR & $5^{\prime}$ & AGUUCUAGAAAUUUUGUGCAAUA \\
\hline mt PTEN-3'-UTR & $5^{\prime}$ & AGUUCUAGAAAUUUUCUCCCUAAA \\
\hline
\end{tabular}

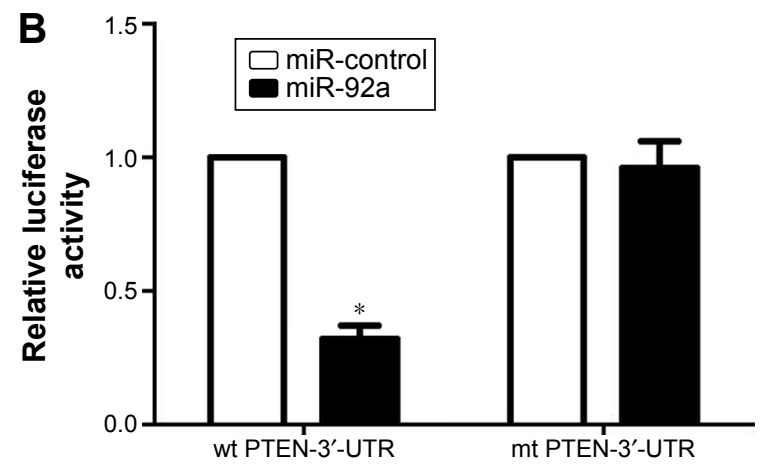

Figure 4 miR-92a interacts with the $3^{\prime}$-UTR of PTEN.

Notes: (A) miR-92a and its predicted complementary sequences in the $3^{\prime}-$ UTR of PTEN. The mutant miR-92a binding sites were generated in the seed region of miR-92a. (B) Overexpression of miR-92a significantly inhibited the luciferase activity that carried wt $3^{\prime}$-UTR of PTEN but had no obvious effect on mt $3^{\prime}-$ UTR of PTEN; $n=3$ independent repeats with similar results; $* P<0.05$.

Abbreviations: miR-92a, microRNA-92a; mt, mutant; PTEN, phosphatase and tensin homolog; 3'-UTR, 3'-untranslated region; wt, wild type.

A
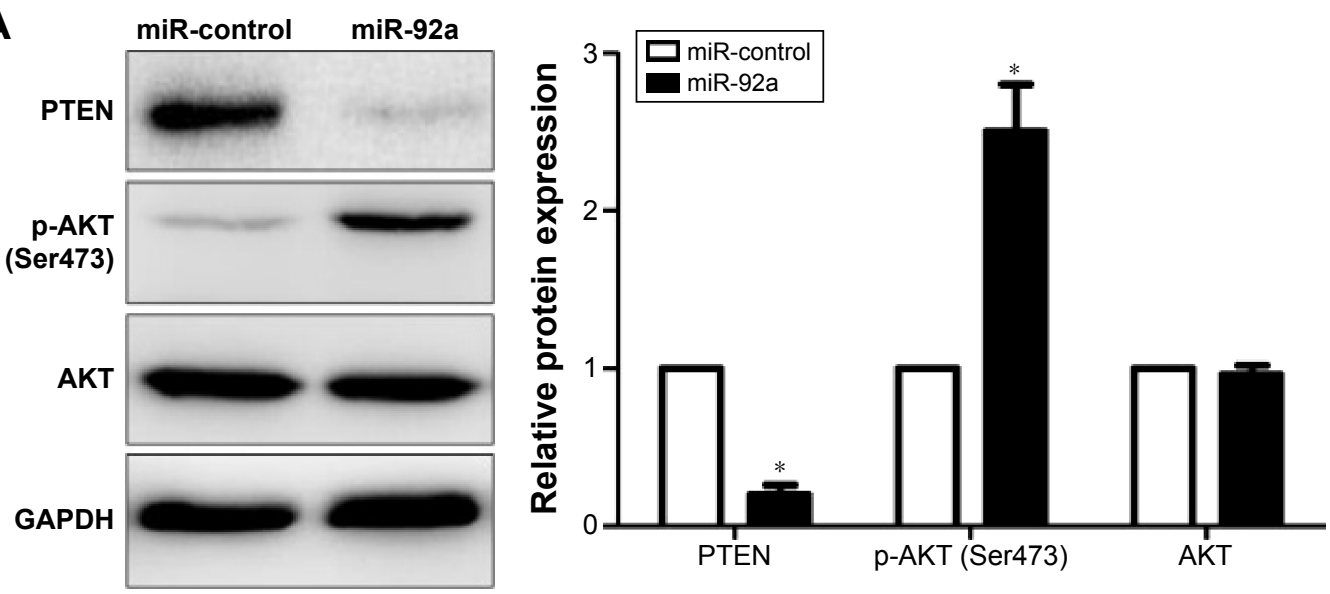

B
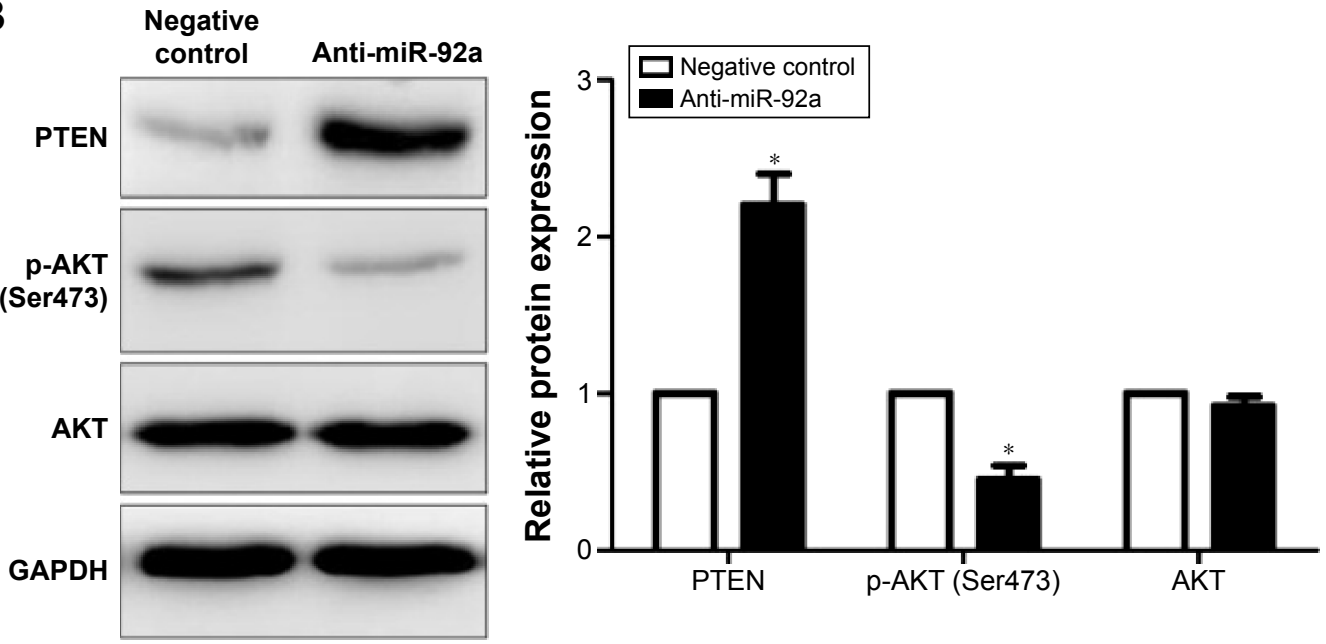

Figure 5 miR-92a regulates the expression of PTEN and the phosphorylation of AKT in NPC cells.

Notes: (A) Overexpression of miR-92a significantly downregulated the level of PTEN and $p-A K T$ in $6-10 B$ cells; $n=3$ independent repeats with similar results; * $P<0.05$. (B) Inhibition of miR-92a resulted in obviously increased level of PTEN and $\mathrm{p}-\mathrm{AKT}$ in 5-8F cells. $\mathrm{n}=3$ independent repeats with similar results; $* P<0.05$.

Abbreviations: GAPDH, glyceraldehyde 3-phosphate dehydrogenase; miR-92a, microRNA-92a; NPC, nasopharyngeal carcinoma; PTEN, phosphatase and tensin homolog. 
the phosphorylation of AKT, which was inhibited by PTEN, was increased $(P<0.05$, Figure 5A). On the other side, the expression of PTEN was significantly increased after miR-92a inhibitors transfection in 5-8F cells $(P<0.05$, Figure 5B), and p-AKT level was obviously decreased $(P<0.05$, Figure $5 \mathrm{~B})$. Taken together, these data suggest that PTEN is a direct downstream target of miR-92a in NPC cells.

\section{miR-92a promotes the metastasis of NPC cells by targeting PTEN/AKT pathway}

To further clarify whether PTEN mediates the functional effects of miR-92a on NPC cells, PTEN expression vector was transfected into miR-92a overexpressing 6-10B cells (6-10B-miR-92a cells). WB results showed that PTEN vector significantly increased PTEN expression and decreased the level of p-AKT in 6-10B-miR-92a cells ( $P<0.05$, Figure 6A). Functionally, overexpression of PTEN partly abrogated the promoting effects of miR-92a on the migration and invasion of 6-10B cells $(P<0.05$, respectively, Figure $6 \mathrm{~B})$. On the other aspect, PTEN siRNA were transfected into 5-8F cells with miR-92a inhibitor transduction (5-8F-anti-miR-92a cells). WB results showed that PTEN siRNA led to a significant inhibition of PTEN expression $(P<0.05$, Figure 7A) and resulted in an obvious increased level of p-AKT $(P<0.05$, Figure 7A) in 5-8F-anti-miR-92a cells. Downregulation of PTEN partly reversed the inhibitory effects of miR-92a on $5-8 \mathrm{~F}$ cell migration and invasion $(P<0.05$, respectively, Figure 7B). These data suggest that miR-92a exerts its effects on migration and invasion of NPC cells by modulating PTEN/AKT pathway.

A
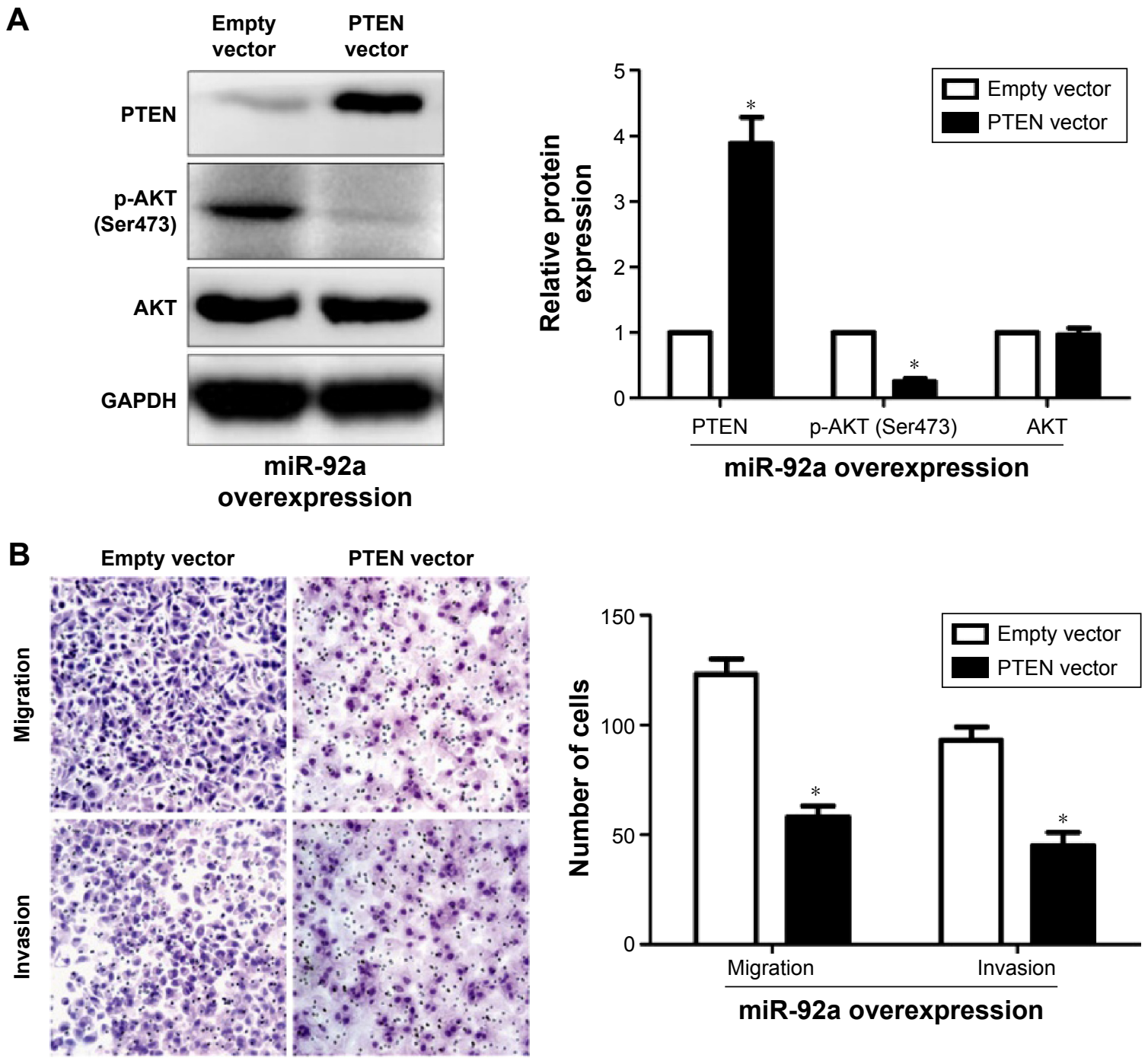

Figure 6 PTEN overexpression abrogates the prometastatic effects of miR-92a mimics on 6-10B cells.

Notes: (A) PTEN expression vector significantly increased the PTEN expression and decreased $\mathrm{p}-\mathrm{AKT}$ expression in miR-92a overexpressing 6-I0B cells; $\mathrm{n}=3$ independent repeats with similar results; $* P<0.05$. (B) Restoration of PTEN in miR-92a overexpressing $6-10 B$ cells abrogated the effects of miR-92a mimics on cell migration and invasion; $\mathrm{n}=3$ repeats with similar results; $* \mathrm{P}<0.05$.

Abbreviations: GAPDH, glyceraldehyde 3-phosphate dehydrogenase; miR-92a, microRNA-92a; PTEN, phosphatase and tensin homolog. 

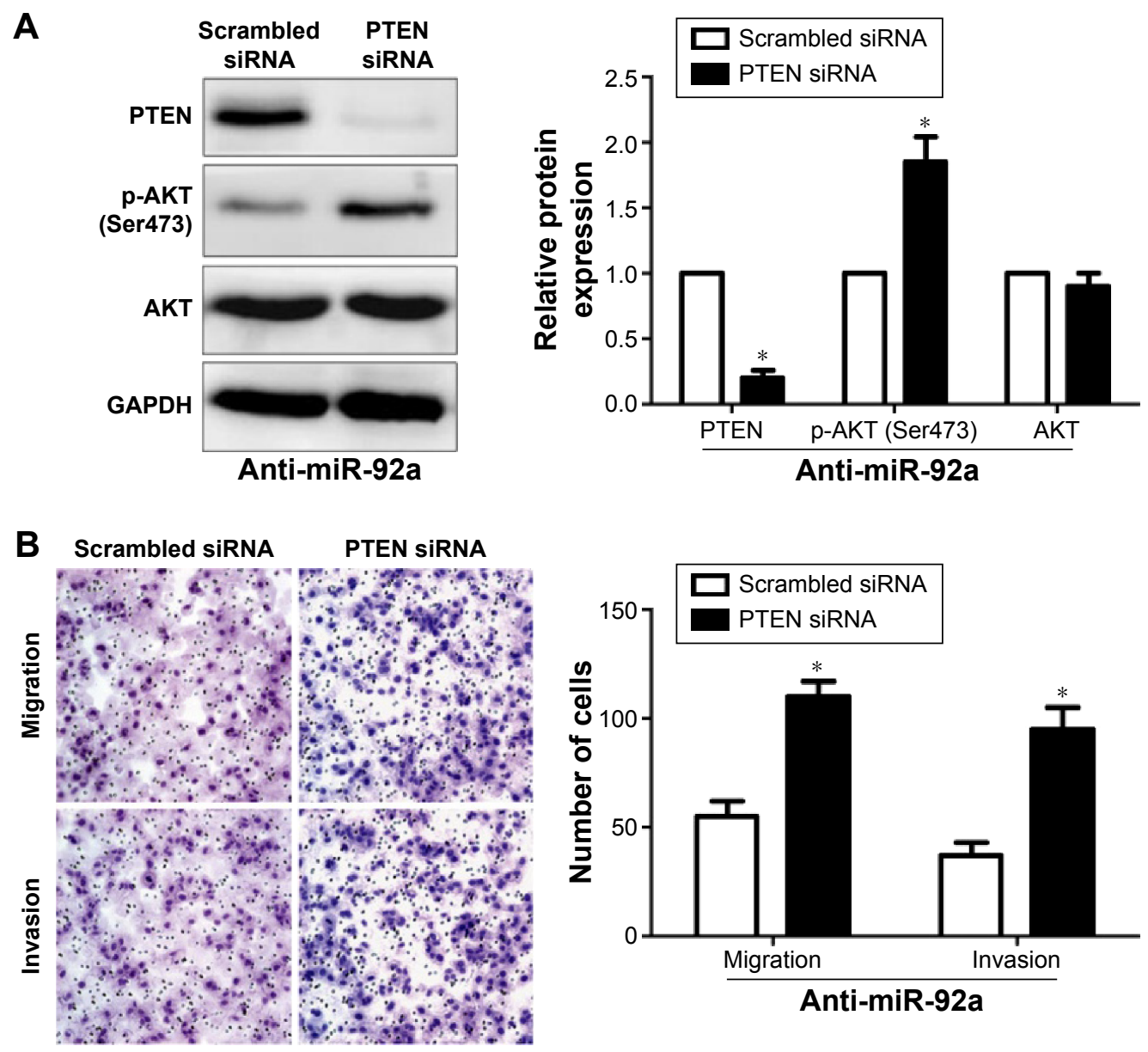

Figure 7 PTEN suppression reverses the inhibitory effects of miR-92a inhibitor on 5-8F cell mobility.

Notes: (A) PTEN siRNA significantly decreased the PTEN expression and increased p-AKT expression in miR-92a downregulating 5-8F cells; $\mathrm{n}=3$ independent repeats with similar results; $* P<0.05$. (B) Suppression of PTEN in miR-92a downregulating 5-8F cells reversed the inhibitory effect of miR-92a inhibitor on cell migration and invasion; $\mathrm{n}=3$ repeats with similar results; $* \mathrm{P}<0.05$.

Abbreviations: GAPDH, glyceraldehyde 3-phosphate dehydrogenase; miR-92a, microRNA-92a; PTEN, phosphatase and tensin homolog; siRNA, small interfering.

\section{Discussion}

The metastasis of NPC is a critical reason for the unsatisfactory prognosis of patients..$^{20}$ Currently, the molecular mechanisms involved in the metastasis of NPC are still not fully understood. During the last two decades, miRNAs have been confirmed to play an important role in the metastatic process of human cancer. ${ }^{21}$ Therefore, the exploration of novel metastasis-related miRNAs can contribute to the identification of novel biomarkers and effective therapeutic targets for patients with NPC.

Previous studies showed that miR-92a was a prognostic marker and played a critical role in the pathogenesis of human cancers. ${ }^{13-17}$ The study of HCC showed that miR-92a expression was abnormally increased, and promoted the growth of HCC cells. ${ }^{13}$ However, miR-92a inhibited the proliferation and metastasis of ovarian cancer cells. Therefore, the expression level and the functional role of miR-92a vary in different cancer types. In this study, we found that the expression level of miR-92a was significantly increased in NPC tissues and cell lines, suggesting an oncogenic role of miR-92a in NPC. Among these four NPC cell lines, 5-8F had the highest metastatic ability while the metastatic ability of 6-10B was the lowest. Accordingly, the expression level of miR-92a was the highest in $5-8 \mathrm{~F}$ cells and was the lowest in 6-10B cells, indicating that the expression level of miR-92a was positively associated with the metastatic ability of NPC cells. And clinical association analysis showed that increased expression of miR-92a was associated with advanced tumornode-metastasis stage and distant metastasis. Survival analysis showed that miR-92a could serve as a promising biomarker for the prognosis of patients with NPC. Then, we investigated the biological role of miR-92a in NPC cells. 
The results arising from transwell assays showed that overexpression of $\mathrm{miR}-92 \mathrm{a}$ promoted the migration and invasion of 6-10B cells, while suppression of miR-92a inhibited the metastatic behaviors of $5-8 \mathrm{~F}$ cells. These results indicate that miR-92a functions as an oncogenic miRNA by facilitating the metastatic ability of NPC cells.

PTEN is a classical tumor suppressor, and has been found to play an important role in the initiation and progression of NPC. ${ }^{22,23}$ In this study, we confirmed that PTEN was a downstream target of miR-92a, and it was also a functional mediator of miR-92a in NPC cells. First, the complementary sequences of miR-92a were found in the 3'-UTR of PTEN. Second, miR-92a overexpression in NPC cells significantly inhibited the luciferase activity of wt $3^{\prime}$-UTR of PTEN, but had no influence on that of mutant $3^{\prime}$-UTR of PTEN, indicating that miR-92a could interact with the $3^{\prime}$-UTR of PTEN through the complementary sequences. Third, overexpression of miR-92a resulted in decreased expression level of PTEN protein, while suppression of miR-92a led to an increased expression level of PTEN protein. Fourth, the rescue experiments showed that PTEN overexpression could abrogate the promoting effects of miR-92a mimics on the migration and invasion of NPC cells, while PTEN downregulation partly reversed the inhibitory effects of miR-92a inhibitor on the metastatic behaviors of NPC cells. These data indicate that miR-92a regulates PTEN abundance and exerts its effects on NPC cells by modulating the expression of PTEN. PTEN is an important regulator of the PTEN/phosphatidyl inositol 3-kinase/AKT pathway. And our data suggested that the phosphorylation of AKT was also accordingly affected by miR-92a. These indicate that miR-92a exerts its effects on migration and invasion of NPC cells by modulating PTEN/AKT pathway. However, it is necessary to mention that PTEN might not be the only downstream target of miR-92a in NPC cells. Thus, more studies need to be performed in the future to identify more novel downstream targets and pathways of miR-92a in NPC.

In conclusion, this study demonstrates for the first time that the expression of miR-92a is significantly increased in NPC tissues and cells. Elevated level of miR-92a is associated with adverse clinicopathological features and poor prognosis of patients with NPC. Functionally, miR-92a can promote the migration and invasion of NPC cells. Furthermore, this study demonstrates that PTEN/AKT pathway is a direct downstream target of miR-92a in NPC cells, and miR-92a exerts its effects on NPC cells by targeting PTEN/AKT pathway.

\section{Acknowledgment}

This study was supported by a grant from the National Natural Science Foundation of China [no 81271057].

\section{Disclosure}

The authors report no conflicts of interest in this work.

\section{References}

1. Yu MC, Yuan JM. Epidemiology of nasopharyngeal carcinoma. Semin Cancer Biol. 2002;12(6):421-429.

2. Ou SH, Zell JA, Ziogas A, Anton-Culver H. Epidemiology of nasopharyngeal carcinoma in the United States: improved survival of Chinese patients within the keratinizing squamous cell carcinoma histology. Ann Oncol. 2007;18(1):29-35.

3. Jia WH, Luo XY, Feng BJ, et al. Traditional Cantonese diet and nasopharyngeal carcinoma risk: a large-scale case-control study in Guangdong, China. BMC Cancer. 2010;10:446.

4. Lai S-Z, Li W-F, Chen L, et al. How does intensity-modulated radiotherapy versus conventional two-dimensional radiotherapy influence the treatment results in nasopharyngeal carcinoma patients? Int J Radiat Oncol Biol Phys. 2011;80(3):661-668.

5. Xiao WW, Huang SM, Han F, et al. Local control, survival, and late toxicities of locally advanced nasopharyngeal carcinoma treated by simultaneous modulated accelerated radiotherapy combined with cisplatin concurrent chemotherapy: long-term results of a phase 2 study. Cancer. 2011;117(9):1874-1883.

6. Bartel DP. MicroRNAs: genomics, biogenesis, mechanism, and function. Cell. 2004;116(2):281-297.

7. Kloosterman WP, Plasterk RH. The diverse functions of microRNAs in animal development and disease. Dev Cell. 2006;11(4):441-450.

8. Mendell JT. MicroRNAs: critical regulators of development, cellular physiology and malignancy. Cell Cycle. 2005;4(9):1179-1184.

9. Tu K, Liu Z, Yao B, Han S, Yang W. MicroRNA-519a promotes tumor growth by targeting PTEN/PI3K/AKT signaling in hepatocellular carcinoma. Int J Oncol. 2016;48(3):965-974.

10. Calin GA, Croce CM. MicroRNA signatures in human cancers. Nat Rev Cancer. 2006;6(11):857-866.

11. Jansson MD, Lund AH. MicroRNA and cancer. Mol Oncol. 2012;6(6): 590-610.

12. Dou C, Wang Y, Li C, et al. MicroRNA-212 suppresses tumor growth of human hepatocellular carcinoma by targeting FOXA1. Oncotarget. 2015;6(15):13216-13228

13. Yang W, Dou C, Wang Y, et al. MicroRNA-92a contributes to tumor growth of human hepatocellular carcinoma by targeting FBXW7. Oncol Rep. 2015;34(5):2576-2584.

14. Ranade AR, Cherba D, Sridhar S, et al. MicroRNA 92a-2*: a biomarker predictive for chemoresistance and prognostic for survival in patients with small cell lung cancer. $J$ Thorac Oncol. 2010;5(8): 1273-1278.

15. Wu CW, Ng SS, Dong YJ, et al. Detection of miR-92a and miR-21 in stool samples as potential screening biomarkers for colorectal cancer and polyps. Gut. 2012;61(5):739-745.

16. Nilsson S, Möller C, Jirström K, et al. Downregulation of miR-92a is associated with aggressive breast cancer features and increased tumour macrophage infiltration. PLoS One. 2012;7(4):e36051.

17. Wang H, Sun Q, Wu Y, et al. Granzyme M expressed by tumor cells promotes chemoresistance and EMT in vitro and metastasis in vivo associated with STAT3 activation. Oncotarget. 2015;6(8): $5818-5831$.

18. Ohyagi-Hara C, Sawada K, Kamiura S, et al. miR-92a inhibits peritoneal dissemination of ovarian cancer cells by inhibiting integrin $\alpha 5$ expression. Am J Pathol. 2013;182(5):1876-1889. 
19. Parsons R, Simpson L. PTEN and cancer. In: El-diery WS, editor. Tumor Suppressor Genes. Totowa, NJ: Humana Press Inc; 2003:147-166.

20. He Q, Ren X, Chen J, et al. miR-16 targets fibroblast growth factor 2 to inhibit NPC cell proliferation and invasion via PI3K/AKT and MAPK signaling pathways. Oncotarget. 2016;7(3):3047-3058.

21. Yates LA, Norbury CJ, Gilbert RJ. The long and short of microRNA. Cell. 2013;153(3):516-519.
22. Cai L, Lyu X, Luo W, et al. EBV-miR-BART7-3p promotes the EMT and metastasis of nasopharyngeal carcinoma cells by suppressing the tumor suppressor PTEN. Oncogene. 2015;34(17):2156-2166.

23. Liang YF, Zeng JC, Ruan JB. Clinical significance of loss expression of PTEN mRNA in nasopharyngeal carcinoma. China Trop Med. 2012;5:020.

\section{Publish your work in this journal}

OncoTargets and Therapy is an international, peer-reviewed, open access journal focusing on the pathological basis of all cancers, potential targets for therapy and treatment protocols employed to improve the management of cancer patients. The journal also focuses on the impact of management programs and new therapeutic agents and protocols on

\section{Dovepress}

patient perspectives such as quality of life, adherence and satisfaction. The manuscript management system is completely online and includes a very quick and fair peer-review system, which is all easy to use. Visit http://www.dovepress.com/testimonials.php to read real quotes from published authors.

Submit your manuscript here: http://www.dovepress.com/oncotargets-and-therapy-journal 